\title{
RELASI GREEN PADA GAMMA-SEMIGRUP SEMIGRUP YANG DIBANGKITKAN DARI SUATU SEMIGRUP
}

\author{
Y.D Sumanto ${ }^{1 *}$, Abdul Aziz ${ }^{2}$, Solikhin ${ }^{3}$, Robertus Heri SU ${ }^{4}$ \\ 1,2,3,4 Departemen Matematika, Universitas Diponegoro \\ Email : ${ }^{1}$ ydsumanto, ${ }^{2}$ abdulaziz, ${ }^{3}$ solikhin, ${ }^{4}$ robertusherisoelisty[@lecturer.undip.ac.id] \\ ${ }^{*}$ Penulis Korespondensi
}

\begin{abstract}
In the $\Gamma$ - semigroup generated from a semigroup, we can define some equivalence relations called the Green relation. Furthermore, we can examine the relationship between the Green relation on the $\Gamma$ - semigroup and the Green relation on its generating semigroup.
\end{abstract}

Keywords: $\Gamma$-semigroup, the Green relation, generating semigroup.

Abstrak. Pada $\Gamma$-semigroup yang dibangkitkan dari suatu semigrup, kita dapat mendefinisikan beberapa relasi ekuivalensi yang disebut relasi Green pada $\Gamma$ - semigrup. Selanjutnya kita dapat mengkaji keterkatitan antara relasi Green pada semigrup pembangkitnya.

Kata kunci: $\Gamma$ - semigrup, relasi Green, semigrup pembangkit.

\section{PENDAHULUAN}

Di dalam [1] telah didefinisikan gagasan tentang $A$ - semigrup yang merupakan generalisasi dari semigrup . Di dalam [2] telah dibahas relasi Green pada suatu semigrup dan beberapa aspek yang terkait. Dalam [3] telah ditunjukkan bahwa setiap semigrup dapat membangkitkan suatu $\Gamma$-semigrup. Sedangkan pembahasan tentang $\Gamma$-semigrup telah diawali dalam [4]. Di dalam [5] dibahas relasi Green pada $\Gamma$-semigrup untuk membangun adjoint semigrup dari suatu $\Gamma-$ semigrup. Dalam jurnal ini, didefinisikan relasi Green pada semigrup yang dibangkitkan dari suatu semigrup dan dikaji keterkaitannya dengan relasi Green pada semigrup pembangkitnya.

\section{RELASI GREEN PADA $\Gamma$-SEMIGRUP}

Misalkan $(S, \alpha)$ suatu semigrup dengan elemen identitas. Relasi Green pada $(S, \alpha)$ didefinisikan dengan

$$
\begin{aligned}
& x L_{\alpha} y \Leftrightarrow S \alpha x=S \alpha y \\
& x R_{\alpha} y \Leftrightarrow x \alpha S=y \alpha S \\
& x J_{\alpha} y \Leftrightarrow S \alpha x \alpha S=S \alpha y \alpha S
\end{aligned}
$$

dan 


$$
\begin{aligned}
& D_{\alpha}=L_{\alpha} \circ R_{\alpha} \\
& H_{\alpha}=L_{\alpha} \cap R_{\alpha}
\end{aligned}
$$

Relasi $L_{\alpha}, R_{\alpha}, J_{\alpha} D_{\alpha}$ dan $H_{\alpha}$ merupakan relasi ekuivalensi. Dalam [2] ditunjukkan bahwa setiap elemen $a \in S$ mendefinisikan operasi biner pada $S$ dengan

$$
\begin{aligned}
x \alpha_{a} y & =(x \alpha a) \alpha y \\
& =x \alpha(a \alpha y)
\end{aligned}
$$

untuk setiap $x, y \in S$.

Jika $\Gamma=\left\{\alpha_{a} \mid a \in S\right\}$ maka $(S, \Gamma)$ merupakan $\Gamma$-semigup, yaitu $\Gamma$-semigrup yang dibangkitkan dari semigrup $(S, \alpha)$. Berikut didefinisikan relasi $L_{\Gamma}, R_{\Gamma}$ dan $J_{\Gamma}$ pada $\Gamma-$ semigrup $(S, \Gamma)$.

Definisi 2.1 Misalkan $(S, \Gamma)$ semigrup yang dibangkitkan dari semigrup $(S, \alpha)$. Pada $\Gamma-$ semigrup $(S, \Gamma)$ didefinisikan relasi $L_{\Gamma}, R_{\Gamma}$ dan $J_{\Gamma}$ sebagai berikut

$$
\begin{aligned}
& x L_{\Gamma} y \Leftrightarrow S \Gamma x=S \Gamma y \\
& x R_{\Gamma} y \Leftrightarrow x \Gamma S=y \Gamma S \\
& x J_{\Gamma} y \Leftrightarrow S \Gamma x \Gamma S=S \Gamma y \Gamma S .
\end{aligned}
$$

Pernyataan - pernyataan di bawah ini ekuivalen dengan Definisi 2.1:

$$
\begin{aligned}
& x L_{\Gamma} y \Leftrightarrow(\exists p, q \in S \wedge \exists \gamma, \delta \in \Gamma)(x \gamma p=y \delta q) \\
& x R_{\Gamma} y \Leftrightarrow(\exists p, q \in S \wedge \exists \gamma, \delta \in \Gamma)(p \gamma x=q \delta y) \\
& x J_{\Gamma} y \Leftrightarrow(\exists p, q, r, s \in S \wedge \exists \gamma, \delta, \mu, v \in \Gamma)(p \gamma x \delta q=r \mu y v s) .
\end{aligned}
$$

Teorema berikut mudah ditunjukkan bahwa $L_{\Gamma}, R_{\Gamma}$ dan $J_{\Gamma}$ merupakan relasi ekuivalensi.

Teorema 2.2 Relasi $L_{\Gamma}, R_{\Gamma}$ dan $J_{\Gamma}$ pada $\Gamma$-semigrup $(S, \Gamma)$ merupakan relasi ekuivalensi.

Proposisi 2.3 Relasi $L_{\Gamma}, R_{\Gamma}$ dan $J_{\Gamma}$ pada $\Gamma-\operatorname{semigrup~}(S, \Gamma)$ memenuhi

$$
\begin{aligned}
& x L_{\Gamma} y \Leftrightarrow(\exists p, q \in S \wedge \exists \gamma, \delta \in \Gamma)(x=p \gamma y \wedge y=q \delta x) \\
& x R_{\Gamma} y \Leftrightarrow(\exists p, q \in S \wedge \exists \gamma, \delta \in \Gamma)(y \gamma p \wedge y=q \delta x) \\
& x J_{\Gamma} y \Leftrightarrow(\exists p, q, r, s \in S \wedge \exists \gamma, \delta, \mu, v \in \Gamma)(x=p \gamma y \delta q \wedge y=r \mu x v s) .
\end{aligned}
$$

Bukti: Akan dibuktikan untuk $L_{\Gamma}$ sedangkan yang lain dengan cara yang sama. Misalkan $x L_{\Gamma} y$ dari Definisi 2.1. maka $S \Gamma x=S \Gamma y$. Karena $S$ memuat elemen identitas misalkan $e$ maka 
$y=e(\alpha e) y=e \alpha_{e} y \in S \Gamma y$. Ini berarti bahwa $y \in S \Gamma x$. Jadi ada $q \in S$ dan $\delta \in \Gamma$ sehingga $y=q \delta x$. Dengan cara yang sama dapat ditunjukkan ada $p \in S$ dan $\gamma \in \Gamma$ sehingga $x=p \gamma y$. Sebaliknya misalkan ada $p, q \in S$ dan $\gamma, \delta \in \Gamma$ sedemikian sehingga $x=p \gamma y, y=q \delta x$, dan

$$
\begin{aligned}
S \Gamma x & =S \Gamma(p \gamma y) \\
& =(S \Gamma p) \gamma y \\
& \subseteq S \Gamma y \\
S \Gamma y & =S \Gamma(q \delta x) \\
& =(S \Gamma q) \delta x \\
& \subseteq S \Gamma x .
\end{aligned}
$$

Jadi $S \Gamma x=S \Gamma y$ berarti $\alpha L_{\Gamma} y$.

Relasi $L_{\Gamma}, R_{\Gamma}$ dan $J_{\Gamma}$ merupakan himpunan pasangan berurutan dari elemen - elemen $S$ sebagai berikut.

$$
\begin{aligned}
& L_{\Gamma}=\left\{(x, y) \in S \times S \mid \alpha L_{\Gamma} y\right\} \\
& R_{\Gamma}=\left\{(x, y) \in S \times S \mid \alpha R_{\Gamma} y\right\} \\
& J_{\Gamma}=\left\{(x, y) \in S \times S \mid \alpha J_{\Gamma} y\right\}
\end{aligned}
$$

Komposisi antara $L_{\Gamma}$ dan $R_{\Gamma}$.bersifat komutatif dan merupakan relasi ekuivalensi.

Proposisi 2.4 Relasi $L_{\Gamma}$ dan $R_{\Gamma}$ memenuhi $L_{\Gamma} \circ R_{\Gamma}=R_{\Gamma} \circ L_{\Gamma}$.

Bukti: Misalkan $(x, y) \in L_{\Gamma} \circ R_{\Gamma}$, maka ada $z \in S$ sehingga $(x, z) \in L_{\Gamma}$ dan $(z, y) \in R_{\Gamma}$. Menurut proposisi 2.3, maka terdapat $p, q, r, s \in S$ dan $\gamma, \delta, \mu, v \in \Gamma$ yang memenuhi $p \gamma x=z$, $q \delta z=x, r \mu z=y$.dan $y v s=z$. Misalkan $z^{\prime}=q \delta z \mu r$, diperoleh $x \mu r=q \delta z \mu r=z^{\prime}$ dan

$$
\begin{aligned}
z^{\prime} v s & =q \delta z \mu r v s \\
& =q \delta y v s \\
& =q \delta z \\
& =x .
\end{aligned}
$$

Ini berarti $\left(x, z^{\prime}\right) \in R_{\Gamma}$. Selanjutnya, $q \delta y=q \delta z \mu r=z^{\prime}$.

$$
\begin{aligned}
p \gamma z^{\prime} & =p \gamma q \delta z \mu r \\
& =p \gamma x \mu r \\
& =z \mu r \\
& =y .
\end{aligned}
$$

Berarti $(x, y) \in R_{\Gamma} \circ L_{\Gamma}$. Jadi diperoleh $L_{\Gamma} \circ R_{\Gamma} \subseteq R_{\Gamma} \circ L_{\Gamma}$. Dengan cara yang sama dapat ditunjukkan bahwa $R_{\Gamma} \circ L_{\Gamma} \subseteq L_{\Gamma} \circ R_{\Gamma}$. Dengan demikian bisa disimpulkan bahwa 
JOURNAL OF FUNDAMENTAL MATHEMATICS

AND APPLICATIONS (JFMA) VOL. 3 NO. 1 (JUN 2020)

Available online at www.jfma.math.fsm.undip.ac.id

$L_{\Gamma} \circ R_{\Gamma}=R_{\Gamma} \circ L_{\Gamma}$. Selanjutnya mudah ditunjukkan bahwa $L_{\Gamma} \circ R_{\Gamma}$ merupakan relasi ekuivalensi, begitu juga irisan antara $L_{\Gamma}$ dan $R_{\Gamma}$ merupakan relasi ekuivalen dan misalkan

$$
\begin{aligned}
& D_{\Gamma}=R_{\Gamma} \circ L_{\Gamma} \\
& H_{\Gamma}=R_{\Gamma} \cap L_{\Gamma} .
\end{aligned}
$$

Berikut ini diberikan teorema yang menggambarkan keterkaitan antara relasi pada $(S, \alpha)$ dengan relasi pada $(S, \Gamma)$.

Teorema 2.5 Jika $x L_{\alpha} y, x R_{\alpha} y$, dan $x J_{\alpha} y$ maka berturut-turut $x L_{\Gamma} y, x R_{\Gamma} y$, dan $x J_{\Gamma} y$.

Bukti: Dibuktikan jika $x L_{\alpha} y$ maka $x L_{\Gamma} y$ sedangkan yang lain sejalan. Misalkan $x L_{\alpha} y$ maka $S \alpha x=S \alpha y$. Diambil sebarang $p \gamma x \in S \Gamma x$ maka ada $c \in S$ sehingga $\gamma=\alpha_{c}$. Selanjutnya,

$$
\begin{aligned}
& p \gamma x=p \alpha_{c} x \\
&=(p \alpha c) \alpha x \\
&=q \alpha x \in S \alpha x \\
& \subseteq S \alpha x \\
&=S \alpha y \\
& \subseteq S \Gamma y
\end{aligned}
$$

Jadi $p \gamma x \in S \Gamma y$ yang berarti $S \Gamma x \subseteq S \Gamma y$. Selanjutnya dengan cara yang sama diperoleh $S \Gamma y \subseteq S \Gamma x$ sehingga $S \Gamma y=S \Gamma x$. Dengan kata lain $x L_{\Gamma} y$.

Contoh 2.1 Diberikan $S=\{a, b, c, d, e, f\}$ dan operasi biner assosiatif $\alpha$ sebagai berikut.

\begin{tabular}{l|llllll}
$\alpha$ & $a$ & $b$ & $c$ & $d$ & $e$ & $f$ \\
\hline$a$ & $a$ & $a$ & $a$ & $a$ & $a$ & $a$ \\
$b$ & $a$ & $b$ & $c$ & $d$ & $e$ & $f$ \\
$c$ & $a$ & $c$ & $c$ & $d$ & $a$ & $a$ \\
$d$ & $a$ & $d$ & $c$ & $d$ & $c$ & $d$ \\
$e$ & $a$ & $e$ & $e$ & $f$ & $a$ & $a$ \\
$f$ & $a$ & $f$ & $e$ & $f$ & $e$ & $f$
\end{tabular}

Dari sini diperoleh relasi $L_{\alpha}$.

$$
\begin{aligned}
& S \alpha a=\{a, b\}=S \alpha b \\
& S \alpha c=\{a, c, e\}=S \alpha e \\
& S \alpha d=\{a, d, f\}=S \alpha f .
\end{aligned}
$$


Sehingga

$$
L_{\alpha}=\{(a, a),(b, b),(c, c),(d, d),(e, e),(f, f),(c, e),(e, c),(d, f),(f, d)\}
$$

Kelas - kelas dari $L_{\alpha}$ adalah

$$
\begin{aligned}
& L_{a}=\left\{x \in S \mid x L_{\alpha} a\right\}=\{a\} \\
& L_{b}=\left\{x \in S \mid x L_{\alpha} b\right\}=\{b\} \\
& L_{c}=\left\{x \in S \mid x L_{\alpha} c\right\}=\{c, e\}=L_{e} \\
& L_{d}=\left\{x \in S \mid x L_{\alpha} d\right\}=\{d, f\}=L_{f}
\end{aligned}
$$

dan $L=\left\{L_{a}, L_{b}, L_{c}, L_{d}\right\}$ sehingga

$$
R_{\alpha}=\{(a, a),(b, b),(c, c),(d, d),(e, e),(f, f),(c, d),(d, c),(e, f), f, e\}
$$

Kelas - kelas dari $R_{\alpha}$ adalah

$$
R_{a}=\{a\}, R_{b}=\{b\}, R_{c}=R_{d}=\{c, d\}, R_{e}=R_{f}=\{e, f\}
$$

dan $R=\left\{R_{a}, R_{b}, R_{c}, R_{d}\right\}$. Relasi $J_{\alpha}$ diperoleh dari

$$
S \alpha a \alpha S=\{a\}, S \alpha b \alpha S=S \alpha c \alpha S=S \alpha d \alpha S=S \alpha e \alpha S=S \alpha f \alpha S=\{a, b, c, d, e, f\}
$$

Sehingga

$$
J_{\alpha}=\left\{\begin{array}{l}
(a, a),(b, b),(b, c),(b, d),(b, e),(b, f),(c, b),(c, c),(c, d),(c, e),(c, f),(d, b),(d, c), \\
(d, d),(d, e),(d, e),(e, b),(e, c),(e, d),(e, f),(f, b),(f, c),(f, d),(f, e),(f, f)
\end{array}\right\}
$$

Kelas - kelas $J_{\alpha}$ adalah

$$
J_{a}=\{a\}, J_{b}=J_{c}=J_{d}=J_{e}=J_{f}=\{b, c, d, e, f\}
$$

dan

$$
J=\left\{J_{a}, J_{b}\right\} .
$$

Sedangkan relasi

$$
\begin{aligned}
& D_{\alpha}=R_{\alpha} \circ L_{\alpha}=J_{\alpha} \\
& H_{\alpha}=R_{\alpha} \cap L_{\alpha}=\{(a, a),(b, b),(c, c),(d, d),(e, e),(f, f)\}
\end{aligned}
$$

Kelas - kelas $H_{\alpha}$ adalah

$$
H=\left\{H_{a}, H_{b}, H_{c}, H_{d}, H_{e}, H_{f}\right\}
$$

dimana setiap kelasnya adalah himpunan singleton. Selanjutnya untuk setiap $u \in S$ didefinisikan operasi biner $\alpha_{u}$ pada $S$ dengan

$$
x \alpha_{u} y=(x \alpha u) \alpha y=x \alpha(u \alpha y)
$$

Sehingga diperoleh $\Gamma=\left\{\alpha_{a}, \alpha_{b}, \alpha_{c}, \alpha_{d}, \alpha_{e}, \alpha_{f}\right\}$ dimana $\alpha_{b}=\alpha$ dan untuk $\left(S, \alpha_{a}\right)$ merupakan semigrup nol dengan $x \alpha_{a} y=a$ untuk setiap $x, y \in S$. Sedangkan untuk $\alpha_{c}, \alpha_{d}, \alpha_{e}$, dan $\alpha_{f}$ diberikan pada tabel berikut. 


\begin{tabular}{|c|c|c|c|}
\hline$\alpha_{c}$ & $\begin{array}{lllll}a & b & c & d & e\end{array}$ & $\alpha_{d}$ & $\begin{array}{lllll}a & b & c & d & e\end{array}$ \\
\hline$a$ & $\begin{array}{llllllll}a & c & c & d & a\end{array}$ & $a$ & $\begin{array}{llllll}a & a & a & a & a & a\end{array}$ \\
\hline$b$ & $\begin{array}{lllllll}a & c & c & d & a\end{array}$ & $b$ & $\begin{array}{lllllll}a & d & c & d & c & d\end{array}$ \\
\hline$c$ & $\begin{array}{llllll}a & c & c & d & a\end{array}$ & $c$ & $\begin{array}{lllllllllllllllll}a & d & c & d & c & d\end{array}$ \\
\hline$d$ & $\begin{array}{lllll}a & c & c & d & a\end{array}$ & $d$ & $\begin{array}{lllll}a & d & c & d & c\end{array}$ \\
\hline$e$ & $a \quad e \quad e f a$ & $e$ & $a f e f e$ \\
\hline$f$ & $a \quad e \quad e f a$ & $f$ & $a f e f e$ \\
\hline
\end{tabular}

\begin{tabular}{|c|c|c|c|c|}
\hline$\alpha_{e}$ & $\begin{array}{llll}a & b & c & d\end{array}$ & $e f$ & $\alpha_{f}$ & $\begin{array}{llllllllll}a & b & c & d & e & f\end{array}$ \\
\hline$a$ & $\begin{array}{llll}a & a & a & a\end{array}$ & $a \quad a$ & $a$ & $\begin{array}{llllll}a & a & a & a & a & a\end{array}$ \\
\hline$b$ & $a e e f$ & $\begin{array}{ll}a & a\end{array}$ & $b$ & $a f e f e f$ \\
\hline$c$ & $\begin{array}{llll}a & a & a & a\end{array}$ & $a \quad a$ & $c$ & $\begin{array}{lllllllllll}a & a & a & a & a & a\end{array}$ \\
\hline$d$ & $a c c c c$ & $a \quad a$ & $d$ & $\begin{array}{lllllll}d & d & c & d & c & d\end{array}$ \\
\hline$e$ & $\begin{array}{llll}a & a & a & a\end{array}$ & $a \quad a$ & $e$ & $\begin{array}{lllllll}a & a & a & a & a & a\end{array}$ \\
\hline$f$ & $a e e f$ & $a \quad a$ & $f$ & $a f e f e f$ \\
\hline
\end{tabular}

Selanjutnya relasi $L_{\Gamma}$ diperoleh dari

$$
\begin{aligned}
& S \Gamma a=\bigcup_{\gamma \in \Gamma} S \gamma a=\{a\} \\
& S \Gamma b=\bigcup_{\gamma \in \Gamma} S \gamma b=S \\
& S \Gamma c=\bigcup_{\gamma \in \Gamma} S \gamma c=\{a, c, e\}=S \Gamma e \\
& S \Gamma d=\bigcup_{\gamma \in \Gamma} S \gamma d=\{a, d, f\}=S \Gamma f
\end{aligned}
$$

Sehingga diperoleh

$$
L_{\Gamma}=\{(a, a),(b, b),(c, c),(d, d),(e, e),(f, f),(c, e),(e, c),(d, f),(f, d)\}
$$

Kelas - kelas dari $L_{\Gamma}$ adalah

dan

$$
L_{a}=\{a\}, L_{b}=\{b\}, L_{c}=L_{e}=\{c, e\}, L_{d}=L_{f}=\{d, f\},
$$

$$
L=\left\{L_{a}, L_{b}, L_{c}, L_{d}\right\}
$$

Sedangkan relasi $R_{\Gamma}$ diperoleh dari

$$
a \Gamma S=\{a\}, b \Gamma S=S, c \Gamma S=d \Gamma S=\{a, c, d\}, e \Gamma S=f \Gamma S=\{a, e, f\} .
$$

Sehingga diperoleh $R_{\Gamma}=\{(a, a),(b, b),(c, c),(d, d),(e, e),(f, f),(c, d),(d, c),(e, f),(f, e)\}$.

Kelas - kelas dari $R_{\Gamma}$ adalah

dan

$$
R_{a}=\{a\}, R_{b}=\{b\}, R_{c}=R_{d}=\{c, d\}, R_{e}=R_{f}=\{e, f\},
$$

$$
R=\left\{R_{a}, R_{b}, R_{c}, R_{d}\right\}
$$


Selanjutnya, relasi $J_{\Gamma}$ diperoleh dari

$$
S \Gamma a \Gamma S=\{a\}, S \Gamma b \Gamma S=S \Gamma c \Gamma S=S \Gamma d \Gamma S=S \Gamma e \Gamma S=S \Gamma f \Gamma S=S .
$$

Sehingga

$$
J_{\Gamma}=\left\{\begin{array}{l}
(a, a),(b, b),(c, c),(d, d),(e, e),(f, f),(b, c),(b, d),(b, e),(b, f), \\
(c, b),(c, d),(c, e),(c, f),(d, b),(d, c),(d, e),(d, f),(e, b),(e, c), \\
(e, d),(e, f),(f, b),(f, c),(f, d),(f, e)
\end{array}\right\} .
$$

Kelas - kelas dari $J_{\Gamma}$ adalah

$$
J_{a}=\{a\}, J_{b}=J_{c}=J_{d}=J_{e}=J_{f}=\{b, c, d, e, f\}, J=\left\{J_{a}, J_{b}\right\}
$$

Selanjutnya, $D_{\Gamma}=R_{\Gamma} \circ L_{\Gamma}=J_{\Gamma}$ dan $H_{\Gamma}=R_{\Gamma} \cap L_{\Gamma}=\{(a, a),(b, b),(c, c),(d, d),(e, e),(f, f)\}$.

Kelas - kelas dari $H_{\Gamma}$ adalah

dan

$$
H_{a}=\{a\}, H_{b}=\{b\}, H_{c}=\{c\}, H_{d}=\{d\}, H_{e}=\{e\}, H_{f}=\{f\},
$$

$$
H=\left\{H_{a}, H_{b}, H_{c}, H_{d}, H_{e}, H_{f}\right\} .
$$

\section{KESIMPULAN}

Berdasarkan pembahasan di atas, diperoleh kesimpulan bahwa pada $\Gamma$-semigroup telah dapat didefinisikan relasi Green dan telah dapat ditunjukkan keterkaitan antara relasi Green pada $\Gamma$-semigrup dengan relasi Green pada semigrup pembangkitnya.

\section{REFERENSI}

[1] Sen, M.K., Saha, N.K., “On Г-Semigroup,” Bull. Calcuta Math. Soc. Vol. 78, pp. 180 186, 1986.

[2] Howie, J.M., Fundamental of Semigroup Theory, Clarendon Press, Oxford, 2003.

[3] Y.D Sumanto, "Grup-Г Dual dari Suatu Grup-Г," Jurnal Matematika, Vol. 15, No 1, pp. 23 $-27,2012$.

[4] Sen, MK., "On $I$-Semigroup," Proc. Of International Conference On Algebra and it's Application, New Delhi, 1984.

[5] Pasko, E., "The adjoint Semigroup of a $\Gamma$-semigroup," Novi Sad J. Math, Vol.17, no. 2 : pp. $31-39,2017$. 